\title{
Augmented reality application to monitor wireless data process in manufacturing operation
}

\begin{abstract}
Advancements are ongoing in digital manufacturing, Human-Machine interfaces are being developed using augmented and virtual reality environments. Manufacturing engineers soon became aware of the use of 3D modelling software and proposed this technique to envisage a virtual representation of machinery and tools being developed for their particular applications. Human visualisation is considered to play an important role in manufacturing. These techniques enable manufacturing engineers to analyse the progress of particular processes and allow them to investigate methods to increase production efficiency. Therefore, particular attention should be focused on the Human-Machine interface to improve its data reliability and productivity, thereby creating a more attractive workplace for future generations. This research presents a review of the different interactions using these types of manufacturing machines with augmented and virtual reality; with simulation and wireless data processing. A brief of statistical study is presented to show the utilisation of these aspects of the technology. A classification of previous related applications is also given pertaining to the tools used and the methods employed. In this review, the different permutations regarding augmented reality and wireless technologies are discussed, it aims to provide a benchmark for potential for manufacturing machine applications and proposes the additional investigation techniques required to improve reliability, faults prediction, power consumption and data processing speed. Further comparisons are discussed to address the gaps that need to be studied too.
\end{abstract}

Keyword: Augmented reality; Manufacturing operation; Monitor and control; Single point incremental process; Wireless 